\title{
Sirtuin 1 Activation Reduces Transforming Growth Factor- $\beta 1-$ Induced Fibrogenesis and Affords Organ Protection in a Model of Progressive, Experimental Kidney and Associated Cardiac Disease
}

Yanling Zhang, Kim A. Connelly, Kerri Thai, Xinglin Wu, Andras Kapus, David Kepecs, and Richard E. Gilbert

Accepted for publication September 13, 2016.

Address correspondence to Richard E. Gilbert, M.D., Ph.D., 61 Queen St. E., Toronto, ON, Canada M5C 2T2. E-mail: richard.gilbert@ utoronto.ca.

\begin{abstract}
Most forms of chronic, progressive kidney disease are characterized by fibrosis whereby the prototypical prosclerotic growth factor, transforming growth factor $\beta$ (TGF- $\beta$ ), is thought to play a pivotal role. With the recent understanding that TGF- $\beta^{\prime}$ s canonical signaling pathway may be modified by acetylation as well as phosphorylation, we explored the role of the $\mathrm{NAD}^{+}$-dependent lysine deacetylase, sirtuin 1 (SIRT1) in fibrogenesis in the cell culture, animal model, and human settings. In vitro, the increase in collagen production that results from TGF- $\beta 1$ stimulation was ameliorated by the allosteric modifier of Sirt1 deacetylase, SRT3025, in association with a reduction in Smad3 reporter activity. In the remnant kidney model (subtotally or $5 / 6$ nephrectomized rats) that develops progressive kidney disease in association with TGF- $\beta$ overexpression, administration of SRT3025 attenuated glomerular filtration rate decline and proteinuria without affecting blood pressure. Glomerulosclerosis and tubulointerstitial fibrosis were similarly reduced with Sirt1 activation as were cardiac structure and function in this rodent model of primary kidney and secondary cardiac disease. Relating these findings to the human setting, we noted a reduction in SIRT1 mRNA in kidney biopsies obtained from individuals with focal glomerulosclerosis. Together these studies highlight the potential of SIRT1 activation as a therapeutic strategy in progressive, fibrotic kidney disease. (Am J Pathol 2017, 187: 80-90; http://dx.doi.org/10.1016/ j.ajpath.2016.09.016)
\end{abstract}

Although not an inevitable consequence of aging, approximately two thirds of individuals experience declining kidney function as they age so that the number of elderly subjects with chronic and end-stage kidney disease continues to rise. ${ }^{1}$ Histologic examination of such kidneys shows so-called agerelated nephrosclerosis that is characterized by glomerulosclerosis, interstitial fibrosis, tubular atrophy, and capillary rarefaction. $^{2}$ These histopathologic features of ostensibly normal aging are also found, albeit in a greatly accelerated form, in a range of chronic kidney diseases (CKDs), including focal glomerulosclerosis whereby the development of fibrosis may be a consequence of activating the profibrotic transforming growth factor $\beta$ (TGF- $\beta$ )/Smad signaling pathway. , $^{3,4}$

Restricting energy intake prolongs life expectancy in yeast, worms, fruit fly, and mammals and also decreasing age- related proteinuria and glomerulosclerosis in rodents. ${ }^{5}$ The discovery that these effects may be mediated by silent information regulator 2 and its mammalian ortholog, the class III lysine deacetylase, sirtuin 1 (SIRT1), has led to the development of specific pharmacologic activators of this enzyme.

Included among the myriad of cell functions that are modulated by SIRT1-dependent deacetylation of lysine

Supported by the Kidney Foundation of Canada grant KFOC120010, the Canadian Institutes of Health Research grant 260193, and Sirtris/Glaxo Smith-Kline (R.G.). R.G. is the Canadian Research Chair in Diabetes Complications, and this research was supported in part by the Canada Research Chair Program.

Disclosure: Sirtris/Glaxo Smith-Kline provided a seed grant (gift of SRT3025) to St. Michael's Hospital. 
residues are pathways that mediate fibrosis. The activity of the prototypical prosclerotic growth factor, TGF- $\beta$, may for instance be modified by SIRT1-mediated deacetylation of certain components of its canonical intracellular signaling pathway. ${ }^{6-8}$ Given the interrelationship between aging, pathologic fibrosis, and Sirt1, we sought to explore its role in kidney fibrosis and associated cardiac dysfunction with human, experimental, and intervention studies, taking advantage of a new generation of potent, specific, and orally bioavailable SIRT1-activating compounds (STACs) that are currently undergoing clinical trial in a range of chronic diseases. ${ }^{9}$

\section{Materials and Methods}

\section{$\left[{ }^{3} \mathrm{H}\right]$-Proline Incorporation Assay}

After serum starvation, rat kidney fibroblasts (NRK-49F) were stimulated with $5 \mathrm{ng} / \mathrm{mL}$ TGF- $\beta 1$ (R \& D Biosystems, Minneapolis, MN) and incubated with $\left[{ }^{3} \mathrm{H}\right]$-proline $(1 \mu \mathrm{Ci} /$ well; PerkinElmer, Waltham, MA) in the presence or absence of SRT3025 (5 $\mu \mathrm{mol} / \mathrm{L}$; gift of James L. Ellis, Glaxo Smith-Kline, King of Prussia, PA) for 44 hours. Incorporation of $\left[{ }^{3} \mathrm{H}\right]$-proline was measured using a liquid scintillation counter (LS 6500 Beckman Instruments; Beckman Coulter, Mississauga, ON, Canada).

\section{Reporter Assay}

Smad3-dependent transcriptional activity was followed by a Smad3-responsive reporter construct containing four consecutive Smad-binding elements (SBEs) driving the expression of firefly luciferase (SBE4-Luc, provided by Anita B. Roberts, NIH, Bethesda, MD), as in our earlier studies. ${ }^{10}$ Briefly, NRK-49F rat kidney fibroblasts were plated $\left(0.15 \times 10^{6}\right.$ cells/well $)$ onto 24 -well plates and after 24 hours were cotransfected with SBE4-Luc and the normalizing vector RL-TK renilla luciferase along with empty vector or dominant negative SIRT1 construct (Flag-SIRT1, H355A; Addgene, Cambridge, MA) or wild-type or acetylationdeficient Smad3 construct (pCMV5/Flag-Smad3 and 378R, respectively) using Lipofectamine 2000 (Life Technologies, Carlsbad, CA) according to the manufacturer's recommendation as previously reported. ${ }^{10}$ After 16 hours, cells were serum starved for 3 hours, then incubated with TGF- $\beta 1$ $(5 \mathrm{ng} / \mathrm{mL})$ with or without SRT3025 $(5 \mu \mathrm{mol} / \mathrm{L})$ for 24 hours after which time cells were lysed, and luciferase activity was determined using the Dual Luciferase Reporter Assay System kit (Promega, Madison, WI) and a luminometer (Lumat 9507; Berthold Technologies, Bad Wildbad, Germany) according to the manufacturer's instructions. For each condition, treatments were performed in duplicates, and experiments were repeated at least three times. From each sample, firefly luciferase activity was normalized to the renilla luciferase activity of the same sample. Results were then expressed as fold changes compared with the mean firefly/renilla ratio of untreated controls.

\section{Smad3 Acetylation}

Kidney cells (HEK-293) were transfected with Smad3 construct (pCMV5B/Flag-Smad3), serum starved, then incubated with TGF- $\beta(5 \mathrm{ng} / \mathrm{mL})$ for 24 hours with or without SRT3025 $(5 \mu \mathrm{mol} / \mathrm{L})$, as described above. Cells were then fractionated into nuclear and cytoplasmic components using a Nuclear/Cytosol Fractionation kit (BioVision, Mountain View, CA) according to the manufacturer's instructions. Detection of acetylated Smad3 was assessed in $200 \mu \mathrm{g}$ of nuclear protein by immunoprecipitating with rabbit anti-Smad3 antibody, followed by Western blot analysis with anti-acetylated lysine antibody (both from Cell Signaling Technology, Beverly, MA).

\section{Animal Study 1}

Sixty-two Fischer 344 rats (Charles River, Montreal, QC, Canada) were maintained at the St. Michael's Hospital Animal Research Vivarium in a temperature-controlled $\left(22^{\circ} \mathrm{C}\right)$ room with ad libitum access to commercial standard rat chow. All animal studies were approved by the St. Michael's Hospital Animal Care Committee in accordance with the Guide for the Care and Use of Laboratory Animals $^{11}$ (NIH Publication No. 85-23, revised 1996). At 8 weeks of age, animals were randomized to one-step subtotal $5 / 6$ nephrectomy (SNX) or sham surgery, as previously reported. ${ }^{12}$ In brief, after anesthesia with $2.5 \%$ isoflurane, the right kidney was removed via subcapsular nephrectomy, and approximately two thirds of the left kidney was infracted via selective ligation of 2 of the 3 or 4 branches of the renal artery. Sham surgery consisted of laparotomy and manipulation of both kidneys before wound closure. One week after surgery, animals were randomized to receive either regular chow or chow containing the Sirt1 activator, SRT3025, then followed for a further 12 weeks. According to their random allocation, the four study groups were sham + vehicle $(n=14)$, sham $+\operatorname{SRT} 3025(n=8)$, SNX + vehicle $(n=18)$, and SNX + SRT3025 $(n=22)$. Every 4 weeks, systolic blood pressure was measured in conscious animals using an occlusive tail-cuff plethysmograph (Powerlab; ADInstruments, Colorado Springs, CO). ${ }^{13}$ In the week before terminations, animals were individually housed in metabolic cages for 24 hours to determine their urinary protein excretion with functional and structural measurements performed on each study animal.

\section{GFR Measurement}

Before sacrifice, rats underwent glomerular filtration rate (GFR) measurement using a modified fluorescein isothiocyanate (FITC)-inulin plasma clearance assay, as reported. ${ }^{13}$ Briefly, rats were injected in the tail vein with $3.74 \mu \mathrm{L} / \mathrm{g}$ body weight FITC-inulin. Tail vein blood was sampled at various time points after FITC-inulin injection. Sample fluorescence was detected using a Fluoroscan Ascent FL machine (Thermo Scientific, Rockford, IL) with settings of 
$485 \mathrm{~nm}$ excitation and $538 \mathrm{~nm}$ emission. GFR was calculated using the following two-phase, exponential decay curve using nonlinear regression statistics as previously described $^{14}$ : GFR $=\mathrm{I} /(\mathrm{A} / \alpha+\mathrm{B} / \beta)$, where $\mathrm{I}$ is the amount of FITC-inulin injected, A and B are the $y$-intercept values for the two decay rates, and $\alpha$ and $\beta$ are the decay constants for the distribution and elimination phases. GFR was reported both in absolute terms and relative to body weight.

\section{Tissue Preparation and Histochemistry}

At the end of the study, rats were terminated with inhaled isoflurane $2.5 \%$ anesthesia. The left renal artery was clamped, and the remnant kidney was removed, decapsulated, and sliced transversely before immersion fixation in $10 \%$ neutral buffered formalin, embedding in cryostat matrix (Tissue-Tek; Sakura, Kobe, Japan), or flash frozen in liquid nitrogen. The heart was also excised and stored in a similar manner. Formalin-fixed tissues were routinely processed, embedded in paraffin, and sectioned before staining histochemically or immunohistochemically as previously described ${ }^{13}$ and detailed below.

\section{Glomerulosclerosis}

Glomerulosclerosis was assessed using a semiquantitative technique in a masked fashion as reported previously. ${ }^{12}$ Briefly, the degree of sclerosis in each glomerulus was quantified on sections stained with periodic acid-Schiff, grading them on a scale of 0 to 4 as follows: grade 0 , normal; grade 1, sclerotic area up to $25 \%$ (minimal); grade 2, sclerotic area $25 \%$ to $50 \%$ (moderate); grade 3 , sclerotic area $50 \%$ to $75 \%$ (moderate to severe); and grade 4 , sclerotic area $75 \%$ to $100 \%$ (severe). A glomerulosclerotic index was then calculated using the following formula: glomerulosclerotic index $=\left(1 \times n_{1}+2 \times n_{2}+3 \times n_{3}+4 \times n_{4}\right) /$ $\left(n_{0}+n_{1}+n_{2}+n_{3}+n_{4}\right)$, where Fi is the percentage of glomeruli in the rat with a given score (i).

\section{Immunohistochemistry}

Immunohistochemistry was performed as previously described. ${ }^{15}$ Deposition of types III and IV collagen were assessed in the tubulointerstitium using goat anti-type III and IV collagen polyclonal antibodies (both from Southern Biotech, Birmingham, AL). The extent of either type III or IV collagen accumulation was quantified in the stained kidney sections using Aperio ImageScope (Leica Biosystems Inc., Wetzlar, Germany) computer-assisted image analysis of ten random nonoverlapping $\times 20$ fields for each animal in a masked fashion as previously described. ${ }^{16}$ Omission of primary antisera served as the negative control.

\section{Assessment of Cardiac Fibrosis}

The accumulation of extracellular matrix within the left ventricle was quantified in Picrosirius red-stained heart sections using Aperio ImageScope (Leica Biosystems Inc.) computer-assisted image analysis of ten random nonoverlapping subendocardial $\times 16$ fields.

\section{Cardiac Catheterization}

Cardiac catheterization and data analysis were performed as previously published. ${ }^{17}$ In brief, after cardiac catheterization with a $2 \mathrm{~F}$ combined conductance catheter-micro-manometer (Model SPR-838; Millar Instruments, Houston, TX), data were acquired under steady state conditions and during preload reduction. The following functional parameters were measured and calculated (Millar analysis software PVAN version 3.6): heart rate, $\mathrm{dp} / \mathrm{dt}^{+}, \mathrm{dp} / \mathrm{dt}^{-}$, Tau logistic, and the slope of the end-diastolic pressure volume relationship (EDPVR).

\section{Gene Expression}

Gene expression was assessed in kidney tissue as previously described. ${ }^{18}$ In brief, SYBR green-based measurement of gene expression were performed on QuantStudio 7 Flex RealTime PCR System (Applied Biosystems, Foster City, CA) according to the manufacturer's instructions using the predesigned sequence-specific primers TGF- $\beta 1$, Sirt1, and Smad3 obtained from Integrated DNA Technologies (Coralville, IA) along with those for rat $\alpha 1$ (IV) collagen, forward 5' ATCCGGCCCTTCATTAGCA-3', reverse 5'-GACTGTGCACCGCCATCA- $3^{\prime}$ and for the housekeeping gene, rRPL13a, forward 5'-GATGAACACCAACCCGTCTC-3', reverse 5'-CACCATCCGCTTTTTCTTGT-3'. Data were analyzed using Applied Biosystems Comparative CT method.

\section{Animal Study 2: Food Intake and Activity}

After the observation that rats that received SRT3025 had lower body weight than their untreated counterparts, a second animal study was conducted to specifically address this issue. Six Fischer 344 rats were randomized to receive either control chow or show containing SRT3025 $(n=3$ per group), as described above, and were studied for 7 days while food intake, body temperature, and activity were measured. In brief, body temperature was measured using an infrared thermometer. After sterile preparation and brief anesthesia, an implantable transmitter (TA-F10; Data Sciences International, St. Paul, MN) was inserted subcutaneously. At least 3 days after implantation, activity was acquired over 72 hours, as previously described. ${ }^{19}$

\section{Biopsy Study}

Renal tissue was obtained from biopsy samples from patients with secondary focal glomerulosclerosis, the clinical data from which have been previously reported. ${ }^{20}$ Control tissue was obtained from 10 patients undergoing donor nephrectomy. Kidney tissue was formalin-fixed and embedded in paraffin before sectioning. All patients gave informed consent, and the study was performed in accordance with the Helsinki Declaration. Total RNA was extracted from biopsy specimens with the Arcturus Paradise Plus Reagent System (Life Technologies, Burlington, ON, Canada). Sirtl gene expression was then 


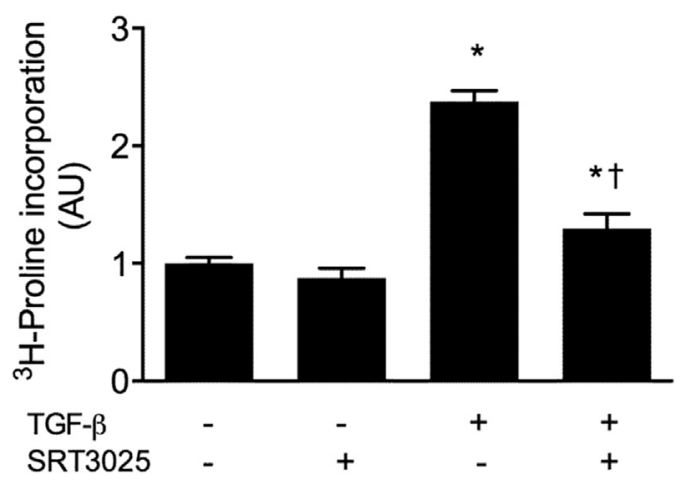

Figure $1 \quad\left[{ }^{3} \mathrm{H}\right]$-proline incorporation. Cultured fibroblasts were preincubated with SRT3025 for 4 hours before stimulation with TGF- $\beta 1$. Without affecting basal $\left[{ }^{3} \mathrm{H}\right]$-proline incorporation, SRT3025 attenuated the augmented incorporation seen with TGF- $\beta . n=3$ per group. ${ }^{*} P<0.05$ versus control; ${ }^{\dagger} P<0.05$ versus TGF- $\beta$. AU, arbitrary unit; TGF- $\beta$, transforming growth factor $\beta$.

measured by quantitative PCR, as described above, using the predesigned sequence-specific primers for human Sirt1 obtained from Integrated DNA Technologies along with those for the housekeeping gene, $h R P L 32 a$, forward 5'-CAACATTGGTTATGGAAGCAACA-3', reverse 5'-TGACGTTGTGGACCAGGAACT-3'.

\section{Statistical Analysis}

Data are expressed as means \pm SEM unless otherwise specified. Because of its skew distribution proteinuria data were logtransformed and expressed as geometric means $\times / \div$ tolerance factor. Between-group differences were analyzed by one-way analysis of variance with Fisher's Protected Least Significant Difference test post hoc. All statistics were performed using GraphPad Prism software version 6 for Mac OS X (GraphPad Software Inc., San Diego, CA). A $P$ value of $<0.05$ was regarded as statistically significant.

\section{Results}

\section{SIRT1 Activation Reduces TGF- $\beta$-Induced Collagen Synthesis}

To determine the potential for SIRT1 activation to attenuate the fibrogenic response to TGF- $\beta$, we exposed rat renal fibroblasts (NRK-49F) to $20 \mathrm{ng} / \mathrm{mL}$ TGF- $\beta 1$ and incubated them with $\left[{ }^{3} \mathrm{H}\right]$-proline for 44 hours to assess collagen synthesis. Compared with the controls, TGF- $\beta 1$ stimulation led to a robust increase in $\left[{ }^{3} \mathrm{H}\right]$-proline incorporation (Figure 1). Pretreatment with SRT3025 had no effect on basal collagen production but reduced TGF- $\beta 1-$ stimulated $\left[{ }^{3} \mathrm{H}\right]$-proline incorporation to a level similar to that of unstimulated cells.

\section{SIRT1 Acetylase Activity Modulates Smad3 Response}

Canonical TGF- $\beta$ downstream signaling involves activation of the regulatory protein, Smad3. Using a Smad3-responsive reporter construct, we examined the effects of the SIRT1 activator, SRT3025, on TGF-induced signal transduction. As anticipated, TGF- $\beta 1$ strongly stimulated SBE4-Luc, the Smad3-responsive promoter construct (Figure 2). Although SRT3025 had no effect on construct activity in the basal state, it prevented TGF- $\beta 1$-induced stimulation.

To ascertain whether the effects of SRT3025 on Smad3 activity were mediated by SIRT1-dependent acetylation, cells were transfected with dominant negative, catalytically inactive mutant SIRT1 (H355A). Diminution of SIRT1 activity in this way led to an expected increase in Smad3 activity in the basal state that was modestly increased with TGF- $\beta 1$ (Figure 2). Moreover, in the presence of the catalytically inactive mutant, SRT3025 was unable to reduce Smad3 activity, indicating that this Sirt1-activating drug requires a catalytically competent Sirt1 enzyme to exert its effects.

\section{SIRT1 Activation Reduces Smad3 Acetylation}

To confirm that SIRT1 activation by SRT3025 deacetylates $S m a d 3$ the abundance of acetylated Smad 3 was assessed in the nuclei of cells exposed to TGF- $\beta$ in the presence of SRT3025. Compared with untreated cells, those that had been incubated with SRT3025 showed less acetylated Smad3 (Figure 3).

\section{SRT3025 Improves Kidney Function and Structure in Experimental CKD}

Using the SNX rat model of chronic progressive kidney disease, we explored the effects of pharmacologic SIRT1 activation on kidney function and structure. This model was chosen because of its resemblance to many forms of CKD in humans, with a progressive reduction in GFR as well as

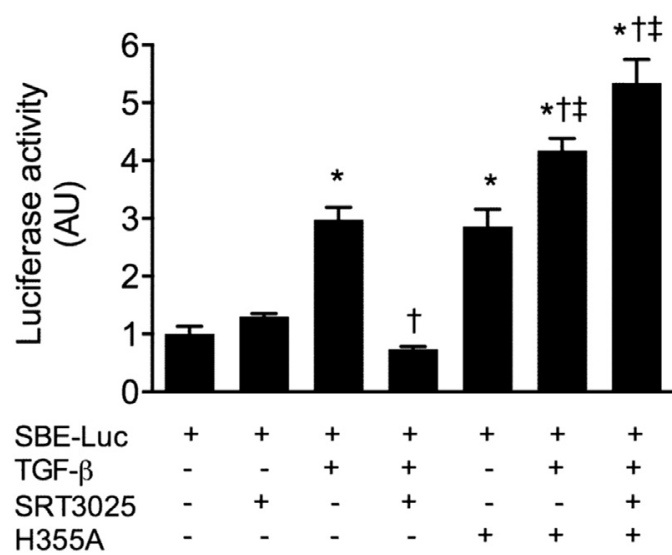

Figure 2 Smad3 reporter activity. Cells transfected with Smad3 reporter construct, SBE4-Luc/pRL-TK, were strongly stimulated by TGF- $\beta 1$. Although SRT3025 had no effect on SBE4-Luc activity in the basal state, it attenuated the response to TGF- $\beta 1$. Cotransfection with a dominant-negative, catalytically inactive mutant Sirt1 (H355A) increased basal reporter activity that was marginally increased further by TGF- $\beta$ with or without SRT3025. $n=3$ per group. ${ }^{*} P<0.05$ versus control; ${ }^{\dagger} P<0.05$ versus TGF- $\beta$; ${ }^{\ddagger} P<0.05$ versus control + H355A. AU, arbitrary unit; TGF- $\beta$, transforming growth factor $\beta$. 
1234

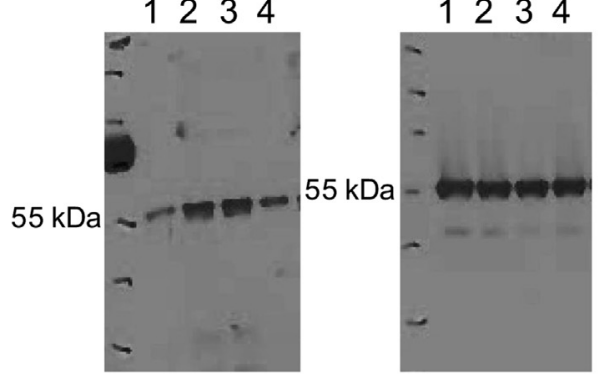

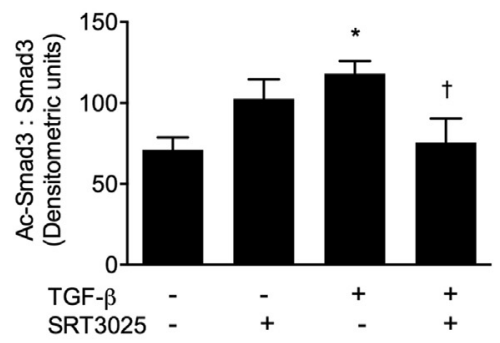

Figure 3 Smad3 acetylation. Nuclear protein extracted from cells transfected with Smad3overexpressing plasmid and stimulated with TGF- $\beta 1$ in the presence of SRT3025 were immunoprecipitated for Smad3 and immunoblotted for acetylated lysine. Ac Smad3 was increased in the presence of TGF- $\beta 1$ but reduced when coincubated with SRT3025. Lane 1, control; lane 2, TGF- $\beta 1$; lane 3, SRT3025; lane 4, TGF$\beta 1+$ SRT3025. $n=3$ per group. ${ }^{*} P<0.05$ versus control; ${ }^{\dagger} P<0.05$ versus TGF- $\beta 1$. Ac, acetylated; TGF- $\beta$, transforming growth factor $\beta$. proteinuria, glomerulosclerosis, and tubulointerstitial disease. Moreover, like CKD in humans, the SNX rat also develops heart failure and responds favorably to blockade of the renin-angiotensin system.

At 12 weeks after 5/6 nephrectomy, 29 of the initial 40 animals were alive (SNX + vehicle, $n=13$; SNX + vehicle, $n=16$ ). All sham animals survived until 12 weeks. Untreated SNX animals displayed a marked reduction in GFR, heavy proteinuria, and hypertension compared with rats that had undergone sham surgery (Figure 3). These functional changes were mirrored in structure whereby no significant pathology was seen in sham animals, whereas the kidneys of partially renal-ablated rats showed extensive glomerulosclerosis and interstitial fibrosis, as evidenced by expansion of periodic acid-Schiff-positive material and immunostainable type III and IV collagen, respectively (Figure 4). A marked attenuation of structural injury was evident in SNX rats that had received SRT3025 with less glomerulosclerosis and interstitial fibrosis (Figure 4). Compared with untreated SNX rats, functional injury was also less evident among nepherectomized rats that had received SRT3025. Without affecting blood pressure, GFR was improved with SRT3025 in SNX rats (Table 1) as was proteinuria with daily urinary protein excretion.

\section{Gene Expression}

Compared with sham surgery kidneys, those of rats that had undergone SNX displayed a marked increase in TGF- $\beta 1$ mRNA that was unaffected by SRT3025 (Figure 5). Collagen IV mRNA that was similarly increased in SNX was reduced in animals that had received SRT3025. A trend toward a reduction in Sirt1 mRNA in SNX versus sham-operated rats was evident, but this did not reach the predefined level of significance but did so when sham-operated rats were compared with those SNX rats that had received SRT3025. For Smad3, no between-group differences were apparent.

\section{SRT3025 Improves Cardiac Function and Structure in Experimental CKD}

Cognizant of the close association between CKD and heart failure along with its negative impact on prognosis, we examined cardiac function in SNX rats with and without SRT3025, focusing particularly on diastolic function. SNX rats demonstrated substantial impairment of diastolic function as demonstrated by an elevated left ventricular EDPVR, a marker of impaired left ventricular compliance that improved with SRT3025 administration (Table 2 and Figure 6).

With cardiac fibrosis as one of the primary determinants of impaired left ventricular relaxation, we examined the magnitude of cardiac matrix accumulation. As in the kidney, SNX lead to significant interstitial fibrosis in the heart that was significantly attenuated by pharmacologic SIRT1 activation (Figure 7).

\section{Food Intake and Activity}

Animals in study 1 that received SRT3025 had lower body weights than their untreated counterparts. To investigate this further, a second study (animal study 2) was undertaken. Although body temperature and food intake were unaffected by SRT3025, activity was higher in animals that had received the Sirt1 activator (Figure 8).

\section{Human FSGS}

As a prototypical fibrotic kidney disease that is characterized by TGF- $\beta$ overexpression, ${ }^{21}$ we explored Sirt1 gene expression in a previously described set archival formalin-fixed paraffin-embedded biopsy tissue ${ }^{20}$ that had been obtained from six samples from patients with biopsy-proven and clinically correlated secondary focal segmental glomerulosclerosis (FSGS) along with kidney tissue samples from 10 living donors. As previously reported, ${ }^{20}$ patients with FSGS were all hypertensive with an elevated urine protein excretion, two of whom were also diagnosed with diabetes mellitus. All donor subjects were normotensive and nondiabetic with normal renal function. SIRT1 gene expression in kidney biopsy tissue from patients with FSGS was less than half that found in healthy donor control subjects (Figure 9).

\section{Discussion}

Following on from the seminal 1930s observation that restricting energy intake extends life span, ${ }^{22}$ the finding that 

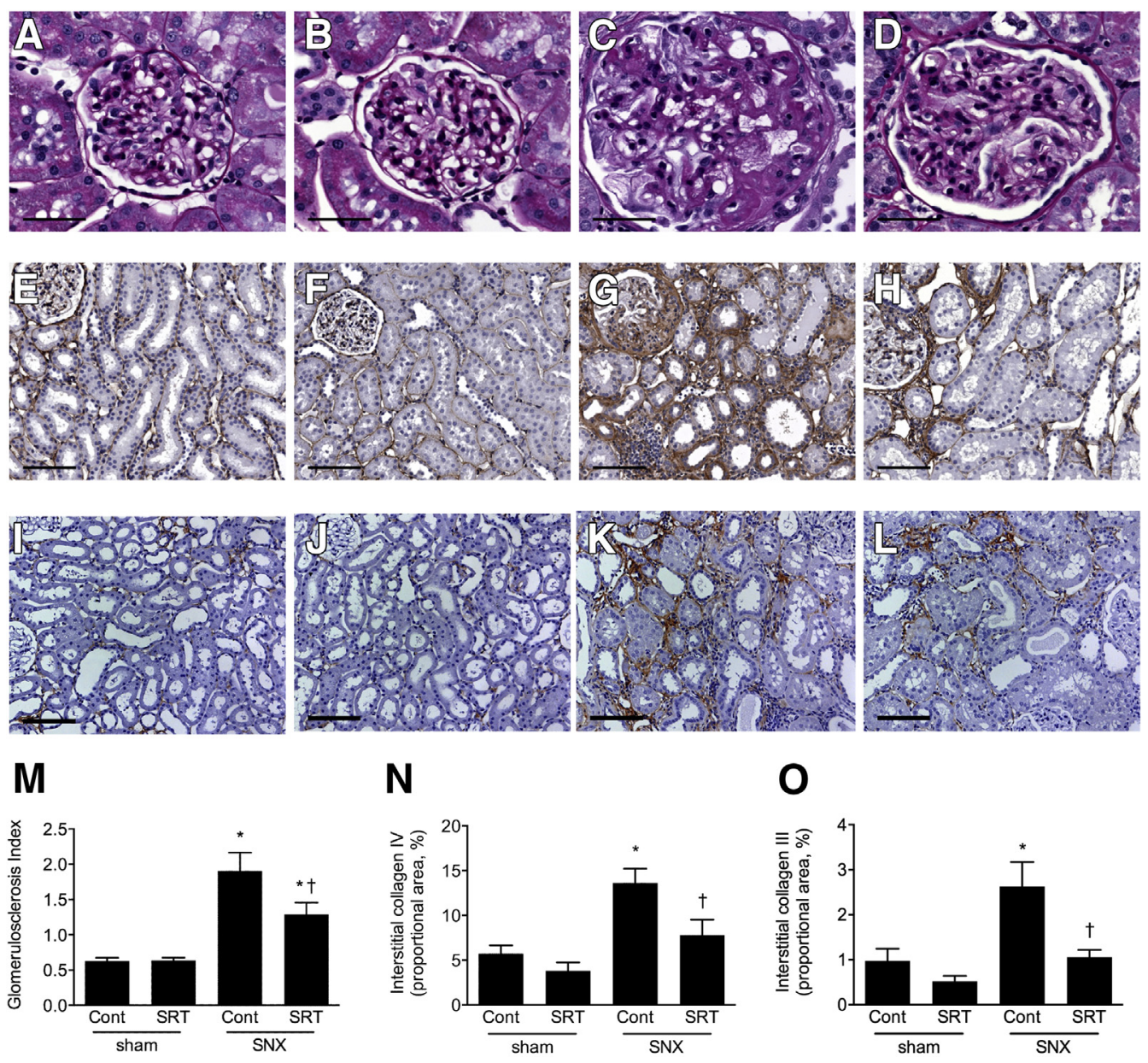

Figure 4 Kidney structure. A-D: To evaluate the extent of glomerulosclerosis, kidney sections were stained with PAS. Representative photomicrographs from sham and SNX rats treated with vehicle (A and C) or SRT3025 (B and D). E-H: To determine the extent of interstitial fibrosis, sections were immunolabeled with anti-collagen type III and type IV antibodies. Representative photomicrographs showing interstitial collagen type IV deposition from sham (E and $\mathbf{F}$ ) or SNX ( $\mathbf{G}$ and $\mathbf{H}$ ) rats treated with vehicle (E and $\mathbf{G})$ or SRT3025 (F and $\mathbf{H})$. I-L: Interstitial type III collagen deposition is shown in sham (I and $\mathbf{J}$ ) or SNX (K and $\mathbf{L}$ ) rats treated with vehicle (I and $\mathbf{K}$ ) or SRT3025 (J and $\mathbf{L})$. $\mathbf{M}-\mathbf{0}$ : The extent of glomerulosclerosis (M) and the proportional area that shows types III $(\mathbf{0})$ and IV (N) collagen immunolabeling are displayed graphically. Compared with vehicle-treated SNX rats, those that received SRT3025 showed reduced glomerulosclerosis and interstitial types III and IV collagen deposition. ${ }^{\star} P<0.05$ versus sham animals; ${ }^{\dagger} P<0.05$ versus vehicle-treated SNX. Scale bars: $50 \mu \mathrm{m}(\mathbf{A}-\mathbf{D}) ; 100 \mu \mathrm{m}(\mathbf{E}-\mathbf{L})$. Original magnification: $\times 400$ (A-D); $\times 160$ (E-L). Cont, control; PAS, periodic acid-Schiff; SNX, subtotal 5/6 nephrectomy; SRT, SRT3025.

this effect may be mediated by silent information regulator protein 2 and its mammalian ortholog SIRT1 has galvanized interest in the pathophysiology and potential therapeutic intervention in age-related and degenerative diseases. ${ }^{23}$ With this in mind, in 2003, Hubbard et $\mathrm{al}^{24}$ screened chemical libraries for STACs, identifying resveratrol, a naturally occurring polyphenol that had also been associated with a myriad of other effects. With the use of this agent, several studies have now shown renoprotective effects in a range of kidney disease models, including unilateral ureteric obstruction, subtotal nephrectomy, and diabetic nephropathy. ${ }^{8,25,26}$ The present study, using a new generation of

Table 1 Blood Pressure and Kidney Function Parameters

\begin{tabular}{lcccc}
\hline Parameter & Sham + vehicle & Sham + SRT3025 & SNX + vehicle & SNX + SRT3025 \\
\hline SBP, $\mathrm{mmHg}$ & $119.2 \pm 4.0$ & $120.1 \pm 4.3$ & $193.6 \pm 7.7^{*}$ & $182.1 \pm 7.5^{*^{\dagger}}$ \\
GFR, $\mu \mathrm{L} / \mathrm{min}$ & $3962 \pm 247$ & $2610 \pm 453$ & $862.2 \pm 127^{*}$ & $1326 \pm 127^{*^{\dagger}}$ \\
GFR, $\mu \mathrm{L} / \mathrm{min} / \mathrm{g}$ & $5.70 \pm 0.27$ & $5.28 \pm 0.88$ & $1.63 \pm 0.23^{*}$ & $2.95 \pm 0.25^{*^{\dagger}}$ \\
Urine $\mathrm{P}, \mathrm{mg} / 24 \mathrm{~h}$ & $18.53 \times / \div 1.1$ & $17.18 \times / \div 1.1$ & $320.1 \times / \div 1.2^{*}$ & $153.5 \times / \div 1.2^{*^{\dagger}}$ \\
\hline
\end{tabular}

${ }^{*} P<0.01$ versus sham + vehicle group.

${ }^{\dagger} P<0.01$ versus SNX + vehicle group.

GFR, glomerular filtration rate; SBP, systolic blood pressure; SNX, subtotal 5/6 nephrectomy; Urine $P$, urine protein (expressed as geometric means $\times / \div$ tolerance factor). 

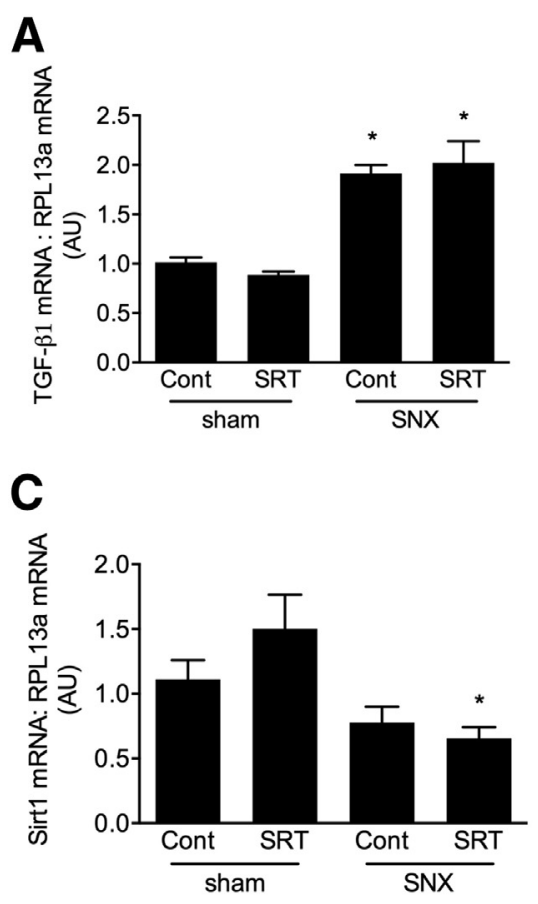

\section{B}
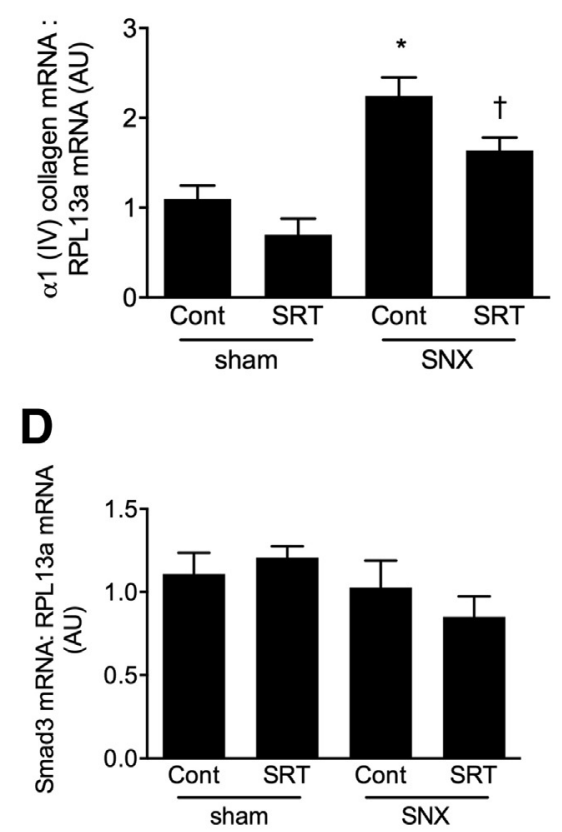

Figure 5 Gene expression. Gene expression of TGF- $\beta 1$ (A), $\alpha 1$ (IV) collagen (B), Sirt1 (C), and Smad3 (D) were assessed by real-time quantitative PCR relative to the housekeeping gene RPL13a in sham and SNX kidneys from animals that received either SRT3025 or vehicle. Although TGF- $\beta 1$ and $\alpha 1$ (IV) collagen mRNA were both increased in the setting of SNX with a nonsignificant reduction in Sirt1 mRNA, administration of SRT3025 to SNX rats was associated with a reduction in $\alpha 1$ (IV) collagen mRNA, and TGF- $\beta$ and Sirt1 mRNA were unaffected. Smad3 mRNA was unaffected by either SNX or SRT3025. ${ }^{*} P<0.05$ versus sham animals; ${ }^{\dagger} P<0.05$ versus vehicle-treated SNX. AU, arbitrary unit; Cont, control; SNX, subtotal $5 / 6$ nephrectomy; SRT, SRT3025; TGF- $\beta$, transforming growth factor $\beta$. potent allosteric STACs, extends these findings in a number of ways. First, by demonstrating that the ability of SRT3025 to attenuate TGF- $\beta$-induced fibrosis depends on catalytically active SIRT1, the study attests to the role of this $\mathrm{NAD}^{+}$-dependent lysine deacetylase in mediating the observed salutary effects. Second, we show that cardiac dysfunction, a common accompaniment to CKD, was also ameliorated by SRT3025. Finally, to indicate its relevance to the human setting, we further noted a diminution in SIRT1 mRNA in human focal glomerulosclerosis, as also reported in diabetic nephropathy, ${ }^{27}$ raising the possibility of a new therapeutic strategy for these common, fibrotic kidney diseases.

The acetylation and de-acetylation of lysine $\varepsilon$-amino groups through the transfer of an acetyl group from acetylcoenzyme A is catalyzed enzymatically by lysine acetylases and deacetylases, respectively. ${ }^{28}$ Although identified in the regulation of histone function some 40 years ago, acetylation is now appreciated to be far more widespread, rivalling phosphorylation, both in breadth and specificity, as a key regulator of protein function. ${ }^{29,30}$ Included in the numerous targets of this post-translational modification is growth factor signaling whereby acetylation has been shown to modulate the key profibrotic growth factor, TGF- $\beta,{ }^{6}$ by Sirt1-mediated lysine deacetylation of its downstream signaling molecules, the Smads. ${ }^{8,31}$

From a therapeutic perspective, recent investigation has focused on the role of Smad3 acetylation as a potential target for dampening the profibrotic effects of excessive activity in the TGF- $\beta$ pathway. The transactivational activity of Smad3 can, for instance, be stimulated by p300, a transcription factor with intrinsic lysine acetylase activity. ${ }^{7}$ Accordingly, reduction of this acetylation-induced stimulation has centered on blocking the enzymatic activity of p300 with the lysine acetyl-transferase inhibitor, curcumin, and its derivatives. $^{32,33}$ In the present study, we adopted the

Table 2 Animal Characteristic and PV-Loop Parameters

\begin{tabular}{|c|c|c|c|c|}
\hline PV-loop parameter & Sham + vehicle & Sham + SRT3025 & SNX + vehicle & SNX + SRT3025 \\
\hline Body weight, g & $684.4 \pm 15.7$ & $506.3 \pm 11.9^{*}$ & $526.8 \pm 11.6^{*}$ & $448.0 \pm 9.9^{* \dagger}$ \\
\hline $\mathrm{HR}, \mathrm{bpm}$ & $291.2 \pm 10.2$ & $272.5 \pm 8.6$ & $263.6 \pm 14.1$ & $254.5 \pm 9.8^{*}$ \\
\hline Tau, m sec & $7.8 \pm 0.3$ & $10.8 \pm 0.7^{\star}$ & $12.6 \pm 1.0^{*}$ & $10.6 \pm 0.7^{\star}$ \\
\hline EDPVR & $0.02 \pm 0.00$ & $0.03 \pm 0.01$ & $0.04 \pm 0.00^{*}$ & $0.02 \pm 0.00^{\dagger}$ \\
\hline $\mathrm{dP} / \mathrm{dt}_{\max }, \mathrm{mmHg} / \mathrm{s}$ & $7169 \pm 379$ & $7151 \pm 236$ & $7425 \pm 442$ & $7220 \pm 407$ \\
\hline $\mathrm{dP} / \mathrm{dt}_{\min }, \mathrm{mmHg} / \mathrm{s}$ & $-7636 \pm 437$ & $-6930 \pm 658$ & $-7634 \pm 509$ & $-7736 \pm 620$ \\
\hline
\end{tabular}

${ }^{*} P<0.05$ versus sham + vehicle group.

${ }^{\dagger} P<0.05$ versus SNX + vehicle group.

$\mathrm{Bpm}$, beats per minute; $\mathrm{dP} / \mathrm{dt}_{\max }$, maximum rate of pressure change in the ventricle; $\mathrm{dP} / \mathrm{dt}_{\min }$, minimum rate of pressure change in the ventricle; EDPVR, end-diastolic pressure volume relationship; HR, heart rate; PV, pressure volume; SNX, subtotal 5/6 nephrectomy; Tau, isovolumic relaxation constant. 
A

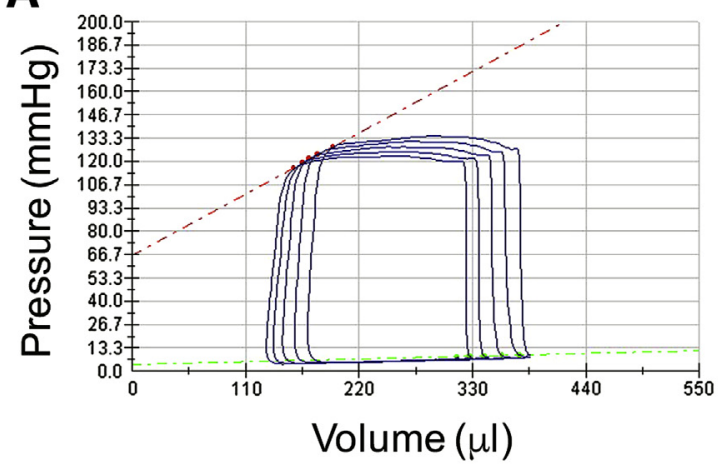

C

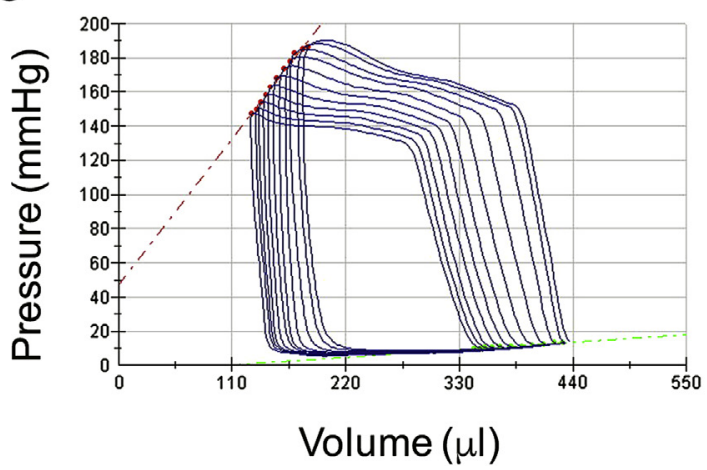

B

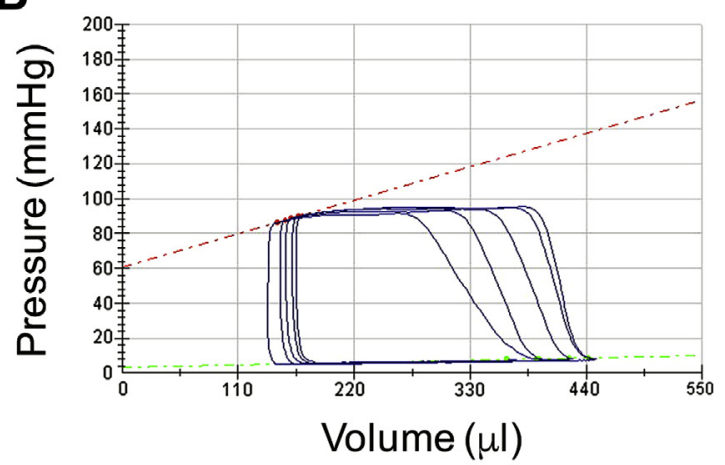

D

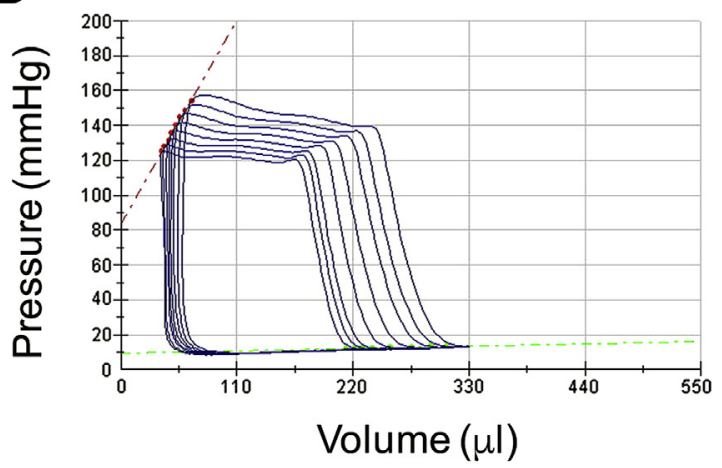

Figure 6 Cardiac function. Twelve weeks after surgery, animals were subjected to invasive cardiac catheterization for cardiac functional analysis with representative pressure-volume loops for sham + vehicle (A), sham + SRT3025 (B), SNX + vehicle (C), or SNX + SRT3025 rats (D). The green line at the lower margin of the pressure-volume loops indicates the LV EDPVR, an index of LV chamber compliance with grouped data provided in Table 2. EDPVR, end-diastolic pressure-volume relationship; LV, left ventricular; SNX, subtotal 5/6 nephrectomy.

alternative approach of stimulating Sirt1-mediated lysine deacetylation of Smad 3, demonstrating that SRT3025 effectively reduced the abundance of acetylated Smad3 in response to TGF- $\beta$ stimulation.

To assess the overall effect of SIRT1 activation on TGF$\beta$-induced fibrogenesis, we noted a reduction in TGF$\beta 1-$ stimulated collagen production and reporter activity by kidney fibroblasts in the presence of SRT3025. To confirm that SRT3025 mediated its effects by stimulating SIRT1mediated deacetylation, we transfected cells with a dominant-negative, catalytically inactive SIRT1 mutant. In contrast to the SRT3025-induced diminution in Smad3 activity that followed incubation with TGF- $\beta$ in the control setting, SRT3025 had no such effect in the presence of the catalytically inactive mutant, H355A, consistent with the activity of STACs, such as SRT3025, as allosteric activators of Sirt $1 .{ }^{24}$ Following on from these cell-based studies, we explored the effects of SRT3025 on the downstream signaling of TGF- $\beta$, focusing on type IV collagen mRNA, given the presence of a Smad-binding element (SBE) in the promoter region of the type IV collagen gene. ${ }^{34}$ Unlike other ostensibly renoprotective agents such as angiotensin converting enzyme inhibitors and angiotensin receptor blockers that suppress TGF- $\beta$ expression in the remnant kidney model, ${ }^{12}$ SRT3025 had no effect on kidney TGF- $\beta$ or Smad3 mRNA. However, consistent with a diminution in
TGF- $\beta$-induced Smad activity, type IV collagen mRNA that was increased in SNX kidneys was reduced with SRT3025.

Fibrosis with its attendant microvascular rarefaction and parenchymal loss is part of the aging process, where with advancing years individuals experience age-associated fibrotic change, manifested by nephrosclerosis with deteriorating kidney function ${ }^{2}$ and cardiac interstitial fibrosis with preserved ejection fraction heart failure. ${ }^{35}$ These ageassociated changes are accelerated in disease states such that most forms of CKD are accompanied by similar fibrotic changes in the interstitial and perivascular regions of the heart in association with preserved ejection fraction heart failure. Consistent with the role of Sirt1 loss in age and disease-associated fibrosis, we found that augmenting the activity of SIRT1 reduced fibrosis in both the hearts and kidneys of animals with CKD.

Cognizant of the controversies surrounding the interpretation of albuminuria reduction as a clinical end point, we focused on glomerular filtration, finding that without affecting blood pressure, SRT3025 improved GFR with a magnitude akin to renin-angiotensin system blockade in this animal model. ${ }^{12}$ Similarly, in light of the importance of loadrelated changes in confounding the interpretation of echocardiography in rodents, ${ }^{17}$ cardiac function was assessed by conductance catheterization, enabling the load-independent 

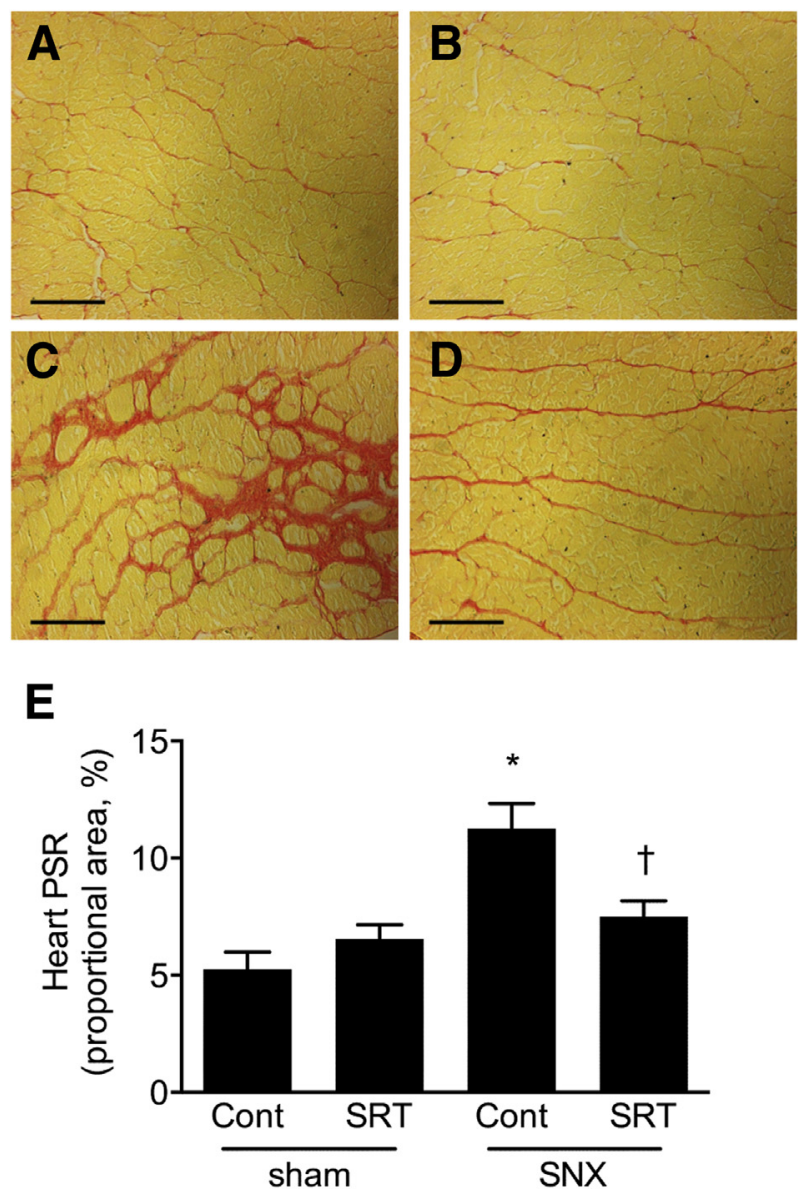

Figure 7 Cardiac structure. Subendocardial heart sections were stained with PSR for assessment of interstitial fibrosis. Representative PSR images for sham + vehicle (A), sham + SRT3025 (B), SNX + vehicle (C), and SNX + SRT3025 (D) along with the quantitative analysis (E). ${ }^{*} P<0.05$ versus sham-operated animals. ${ }^{\dagger} P<0.05$ versus $S N X+$ vehicle. Scale bar $=$ $100 \mu \mathrm{m}(\mathbf{A}-\mathbf{D})$. Original magnification, $\times 160$ (A-D). Cont, control; PSR, Picrosirius red; SNX, subtotal 5/6 nephrectomy; SRT, SRT3025.

acquisition of pressure and volume measurements. Using this method, we noted diastolic dysfunction in SNX rats, as in humans with CKD, with an increase in the EDPVR that was substantially attenuated by SRT3025.

Although the findings of the present study align with several other reports showing deacetylase activation attenuates TGF- $\beta$-induced signaling and fibrosis, ${ }^{8,31,36}$ the reverse

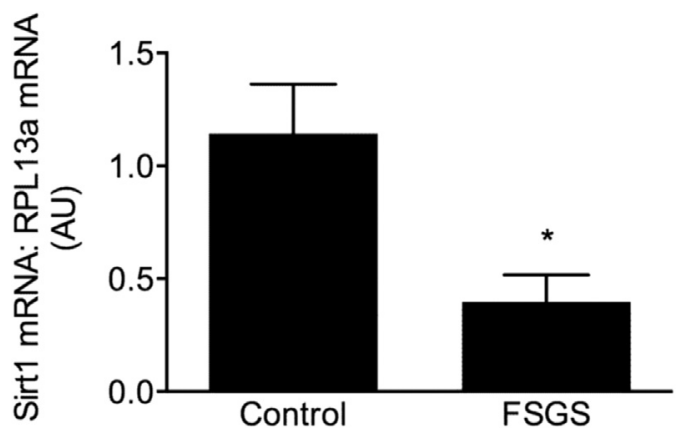

Figure 9 Sirt1 mRNA in human kidney biopsies. Expression of Sirt1 mRNA was assessed by real-time quantitative PCR using RPL13a as a control for loading and expressed as AUs. Compared with donor controls Sirt1 mRNA derived from patients with secondary FSGS was markedly reduced. ${ }^{*} P<0.05$. AU, arbitrary unit; FSGS, focal segmental glomerulosclerosis.

has also been reported whereby inhibition of a deacetylase, HDAC6, similarly reduced fibrogenesis. ${ }^{37,38}$ Such findings indicate the complexities of regulation by acetylation that remain to be unraveled, reflecting not only the range of substrates for the numerous lysine acetylases and decatylases but also which lysine residues within a specific protein may be subject to this post-translational modification. Although it is evident that the function of various proteins can be modified by both acetylation and deacetylation, the summation of their effects is not easily predicted, underscoring the importance of testing in the in vivo setting.

Treatment with SRT3025 was associated with lower body weight compared with untreated animals. Investigating this in a further animal study, food intake and body temperature were unaffected by Sirt1 activation although activity was measurably increased. These effects are consistent with the previously documented role of Sirt1 in mediating the increase in activity associated with caloric restriction, ostensibly by changes in hypothalamic function. ${ }^{39,40}$ The effects of Sirt1 activation are, however, tempered by genetic background with obese, diabetic $\mathrm{db} / \mathrm{db}$ mice responding to Sirt1 activation with a diminution in physical activity. ${ }^{18}$

This study has a number of limitations. Although Smad acetylation and activity were reduced by Sirt 1 activation, the reduction in fibrosis and improvements in kidney and heart function may not necessarily be a direct effect of SRT3025 on Smad-dependent signaling. Indeed, Sirt1-mediated
A

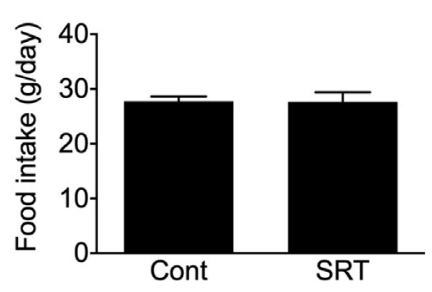

B

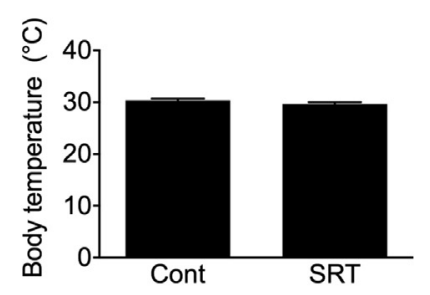

\section{C}

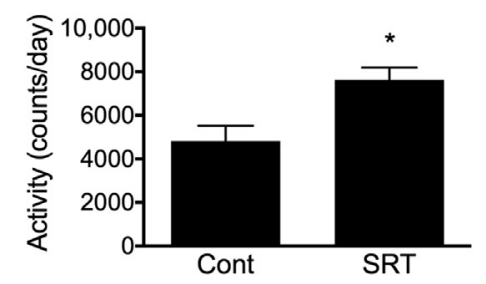

Figure 8 Animal study 2. Fischer rats, treated with or without SRT3025 were studied over 10 days. Although food intake (A) and body temperature (B) were unaffected by Sirt1 activation, administration of SRT3025 led to an increase in physical activity (C). ${ }^{*} P<0.05$ versus control animals. Cont, control; SRT, SRT3025. 
acetylation is not confined to the Smad pathway, so that the beneficial effects observed may be indirect and a consequence of the acetylation of other proteins such as histones. Similarly, although we have focused on fibrogenesis, other key mediators of kidney injury such as NF- $\kappa \mathrm{B}$-induced inflammatory pathways may have also been affected. Moreover, although Sirt1 activation led to improvements in structure and function in both the kidney and heart, suggesting that both organs were directly affected, it is also possible that, alternatively, the reductions in cardiac injury may rather have been a consequence of improved kidney function or vice versa. Although the remnant kidney model shares many functional and structural features with human FSGS, the magnitude of SRT1 reduction in SNX rat kidneys was numerically less than in FSGS biopsies and, although trending, did not reach statistical significance. Finally, as with any experimental study, the ability to extrapolate the findings to the human setting is fraught; although we were able to examine Sirt1 gene expression in biopsy specimens from patients with FSGS, the number and paucity of tissues precluded other analyses being undertaken.

\section{Conclusions}

The reduction in SIRT1 mRNA in human focal glomerulosclerosis biopsies, the effects of SIRT1 activation on TGF- $\beta$ signaling, and the ability of pharmacologic SIRT1 activation to ameliorate fibrosis and to attenuate organ dysfunction in an animal model of advanced kidney disease with concomitant cardiac dysfunction together highlight the potential utility of this strategy, the marked differences between animal models and human disease notwithstanding.

\section{Acknowledgments}

We thank Jennifer Switzer for her technical assistance with the animal studies and Suzanne Advani for her assistance with histology.

\section{References}

1. Choudhury D, Levi M: Kidney aging-inevitable or preventable? Nat Rev Nephrol 2011, 7:706-717

2. Rule AD, Amer H, Cornell LD, Taler SJ, Cosio FG, Kremers WK, Textor SC, Stegall MD: The association between age and nephrosclerosis on renal biopsy among healthy adults. Ann Intern Med 2010, 152:561-567

3. Kim JH, Kim BK, Moon KC, Hong HK, Lee HS: Activation of the TGF-beta/Smad signaling pathway in focal segmental glomerulosclerosis. Kidney Int 2003, 64:1715-1721

4. Ruiz-Torres MP, Bosch RJ, O’Valle F, Del Moral RG, Ramirez C, Masseroli M, Perez-Caballero C, Iglesias MC, Rodriguez-Puyol M, Rodriguez-Puyol D: Age-related increase in expression of TGF-beta1 in the rat kidney: relationship to morphologic changes. J Am Soc Nephrol 1998, 9:782-791

5. Choudhury D, Levi M, Tuncel M: Aging and kidney disease. Edited by Taal MW, Chertow GM, Marsden PA, Skorecki K, Yu SL,
Brener BM. In Brenner and Rector's The Kidney. Philadelphia, PA: Elsevier, 2012. pp. 809-841

6. Simonsson M, Kanduri M, Gronroos E, Heldin CH, Ericsson J: The DNA binding activities of $\operatorname{Smad} 2$ and $\mathrm{Smad} 3$ are regulated by coactivator-mediated acetylation. J Biol Chem 2006, 281:39870-39880

7. Inoue $\mathrm{Y}$, Itoh $\mathrm{Y}$, Abe $\mathrm{K}$, Okamoto $\mathrm{T}$, Daitoku $\mathrm{H}$, Fukamizu $\mathrm{A}$, Onozaki K, Hayashi H: Smad3 is acetylated by p300/CBP to regulate its transactivation activity. Oncogene 2007, 26:500-508

8. Li J, Qu X, Ricardo SD, Bertram JF, Nikolic-Paterson DJ: Resveratrol inhibits renal fibrosis in the obstructed kidney: potential role in deacetylation of Smad3. Am J Pathol 2010, 177:1065-1071

9. Hubbard BP, Sinclair DA: Small molecule SIRT1 activators for the treatment of aging and age-related diseases. Trends Pharmacol Sci 2014, 35:146-154

10. Masszi A, Speight P, Charbonney E, Lodyga M, Nakano H, Szaszi K, Kapus A: Fate-determining mechanisms in epithelial-myofibroblast transition: major inhibitory role for Smad3. J Cell Biol 2010, 188:383-399

11. Committee for the Update of the Guide for the Care and Use of Laboratory Animals; National Research Council: Guide for the Care and Use of Laboratory Animals: Eighth Edition. Washington, DC, National Academies Press, 2011

12. Wu L, Cox A, Roe C, Dziadek M, Cooper ME, Gilbert RE: Transforming growth factor $\beta 1$ and renal injury following subtotal nephrectomy in the rat: role of the renin-angiotensin system. Kidney Int 1997, 51:1553-1567

13. Yuen DA, Connelly KA, Zhang Y, Advani SL, Thai K, Kabir G, Kepecs D, Spring C, Smith C, Batruch I, Kosanam H, Advani A, Diamandis E, Marsden PA, Gilbert RE: Early outgrowth cells release soluble endocrine antifibrotic factors that reduce progressive organ fibrosis. Stem Cells 2013, 31:2408-2419

14. Horwitz EM, Gordon PL, Koo WK, Marx JC, Neel MD, McNall RY, Muul L, Hofmann T: Isolated allogeneic bone marrow-derived mesenchymal cells engraft and stimulate growth in children with osteogenesis imperfecta: implications for cell therapy of bone. Proc Natl Acad Sci U S A 2002, 99:8932-8937

15. Advani A, Gilbert RE, Thai K, Gow RM, Langham RG, Cox AJ, Connelly KA, Zhang Y, Herzenberg AM, Christensen PK, Pollock CA, Qi W, Tan SM, Parving HH, Kelly DJ: Expression, localization, and function of the thioredoxin system in diabetic nephropathy. J Am Soc Nephrol 2009, 20:730-741

16. Kelly DJ, Chanty A, Gow RM, Zhang Y, Gilbert RE: Protein kinase Cbeta inhibition attenuates osteopontin expression, macrophage recruitment, and tubulointerstitial injury in advanced experimental diabetic nephropathy. J Am Soc Nephrol 2005, 16:1654-1660

17. Connelly KA, Prior DL, Kelly DJ, Feneley MP, Krum H, Gilbert RE: Load-sensitive measures may overestimate global systolic function in the presence of left ventricular hypertrophy: a comparison with loadinsensitive measures. Am J Physiol Heart Circ Physiol 2006, 290: H1699-H1705

18. Gilbert RE, Thai K, Advani SL, Cummins CL, Kepecs DM, Schroer SA, Woo M, Zhang Y: SIRT1 activation ameliorates hyperglycaemia by inducing a torpor-like state in an obese mouse model of type 2 diabetes. Diabetologia 2015, 58:819-827

19. Garver J, Bermeo-Blanco OA, Gibson N, Bogie H, Grenwis J, Vela EM: Implantation and monitoring of a novel telemetry unit in the Syrian golden hamster model. J Invest Surg 2012, 25:186-196

20. Chen LH, Advani SL, Thai K, Kabir MG, Sood MM, Gibson IW, Yuen DA, Connelly KA, Marsden PA, Kelly DJ, Gilbert RE, Advani A: SDF-1/CXCR4 signaling preserves microvascular integrity and renal function in chronic kidney disease. PLoS One 2014, 9:e92227

21. Iwano M, Akai Y, Fujii Y, Dohi Y, Matsumura N, Dohi K: Intraglomerular expression of transforming growth factor-beta 1 (TGF-beta 1) mRNA in patients with glomerulonephritis: quantitative analysis by competitive polymerase chain reaction. Clin Exp Immunol 1994, 97:309-314

22. McCay CM, Crowell MF, Maynard LA: The effect of retarded growth upon the length of life span and upon the ultimate body size. J Nutr 1935, 10:63-79 
23. Guarente L: Calorie restriction and sirtuins revisited. Genes Dev 2013 , 27:2072-2085

24. Hubbard BP, Gomes AP, Dai H, Li J, Case AW, Considine T, Riera TV, Lee JE, E SY, Lamming DW, Pentelute BL, Schuman ER, Stevens LA, Ling AJ, Armour SM, Michan S, Zhao H, Jiang Y, Sweitzer SM, Blum CA, Disch JS, Ng PY, Howitz KT, Rolo AP, Hamuro Y, Moss J, Perni RB, Ellis JL, Vlasuk GP, Sinclair DA: Evidence for a common mechanism of SIRT1 regulation by allosteric activators. Science 2013, 339:1216-1219

25. Huang XZ, Wen D, Zhang M, Xie Q, Ma L, Guan Y, Ren Y, Chen J, Hao CM: Sirt1 activation ameliorates renal fibrosis by inhibiting the TGF-beta/Smad3 pathway. J Cell Biochem 2014, 115:996-1005

26. Kitada M, Kume S, Imaizumi N, Koya D: Resveratrol improves oxidative stress and protects against diabetic nephropathy through normalization of Mn-SOD dysfunction in AMPK/SIRT1-independent pathway. Diabetes 2011, 60:634-643

27. Chuang PY, Dai Y, Liu R, He H, Kretzler M, Jim B, Cohen CD, He JC: Alteration of forkhead box O (foxo4) acetylation mediates apoptosis of podocytes in diabetes mellitus. PLoS One 2011, 6:e23566

28. Kosanam H, Thai K, Zhang Y, Advani A, Connelly KA, Diamandis EP, Gilbert RE: Diabetes induces lysine acetylation of intermediary metabolism enzymes in the kidney. Diabetes 2014, 63: 2432-2439

29. Patel J, Pathak RR, Mujtaba S: The biology of lysine acetylation integrates transcriptional programming and metabolism. Nutr Metab (Lond) 2011, 8:12

30. Kouzarides T: Acetylation: a regulatory modification to rival phosphorylation? EMBO J 2000, 19:1176-1179

31. Kume S, Haneda M, Kanasaki K, Sugimoto T, Araki S, Isshiki K, Isono M, Uzu T, Guarente L, Kashiwagi A, Koya D: SIRT1 inhibits transforming growth factor beta-induced apoptosis in glomerular mesangial cells via Smad7 deacetylation. J Biol Chem 2007, 282: $151-158$
32. Li HL, Liu C, de Couto G, Ouzounian M, Sun M, Wang AB, Huang Y, He CW, Shi Y, Chen X, Nghiem MP, Liu Y, Chen M, Dawood F, Fukuoka M, Maekawa Y, Zhang L, Leask A, Ghosh AK, Kirshenbaum LA, Liu PP: Curcumin prevents and reverses murine cardiac hypertrophy. J Clin Invest 2008, 118:879-893

33. Bugyei-Twum A, Advani A, Advani SL, Zhang Y, Thai K, Kelly DJ, Connelly KA: High glucose induces Smad activation via the transcriptional coregulator p300 and contributes to cardiac fibrosis and hypertrophy. Cardiovasc Diabetol 2014, 13:89

34. Li Y, Shen Y, Li M, Su D, Xu W, Liang X, Li R: Inhibitory effects of peroxisome proliferator-activated receptor gamma agonists on collagen IV production in podocytes. Mol Cell Biochem 2015, 405:233-241

35. Mohammed SF, Hussain S, Mirzoyev SA, Edwards WD, Maleszewski JJ, Redfield MM: Coronary microvascular rarefaction and myocardial fibrosis in heart failure with preserved ejection fraction. Circulation 2015, 131:550-559

36. Simic P, Williams EO, Bell EL, Gong JJ, Bonkowski M, Guarente L: SIRT1 suppresses the epithelial-to-mesenchymal transition in cancer metastasis and organ fibrosis. Cell Rep 2013, 3:1175-1186

37. Shan B, Yao TP, Nguyen HT, Zhuo Y, Levy DR, Klingsberg RC, Tao H, Palmer ML, Holder KN, Lasky JA: Requirement of HDAC6 for transforming growth factor-beta1-induced epithelial-mesenchymal transition. J Biol Chem 2008, 283:21065-21073

38. Choi SY, Ryu Y, Kee HJ, Cho SN, Kim GR, Cho JY, Kim HS, Kim IK, Jeong MH: Tubastatin A suppresses renal fibrosis via regulation of epigenetic histone modification and Smad3-dependent fibrotic genes. Vascul Pharmacol 2015, 72:130-140

39. Chen D, Steele AD, Lindquist S, Guarente L: Increase in activity during calorie restriction requires Sirt1. Science 2005, 310:1641

40. Boily G, Seifert EL, Bevilacqua L, He XH, Sabourin G, Estey C, Moffat C, Crawford S, Saliba S, Jardine K, Xuan J, Evans M, Harper ME, McBurney MW: SirT1 regulates energy metabolism and response to caloric restriction in mice. PLoS One 2008, 3:e1759 